\title{
Dos registros de atropellamiento de Leopardus wiedii y Herpailurus yagouaroundi (carnivora: felidae) en Yucatán, México
}

\author{
Alvaro Monter-Pozos ${ }^{(1 D}$, Julio C. Hernández-Hernández²* iD \\ 1 SER Social \& Environmental Response. Baja California 278, int. 305, Col. Hipódromo, Del. Cuauhtémoc, C.P. 06100, Ciudad \\ de México, México. \\ 2 Pronatura Veracruz A.C. Ignacio Zaragoza 153, Centro, C.P. 91500, Veracruz, México. \\ *Correspondencia: jhernandez@pronaturaveracruz.org
}

\section{Resumen}

La mortalidad de la fauna silvestre debido a la colisión con vehículos es un problema poco estudiado. En México, se ha incrementado el interés por determinar los efectos negativos y las acciones de mitigación ante el impacto de las redes viales sobre las poblaciones de vertebrados silvestres. En este estudio se documentan dos casos del aparente atropellamiento de un individuo de jaguarundi y uno de tigrillo, en carreteras estatales de la región litoral centro del estado de Yucatán, México. Ambos registros ponen de manifiesto la importancia de estudios que cuantifiquen el atropellamiento de vertebrados en carreteras para planificar su mitigación.

Palabras clave: Atropellos, Conservación, Efecto de las carreteras, Felinos.

\section{Abstract}

The mortality of wildlife due to vehicle collisions is a poorly studied problem. In Mexico, interest in determining the negative effects and mitigation actions regarding the impact of road networks on wild vertebrate populations has increased. This study documents two cases of the apparent run over of an individual of jaguarundi and one of tigrillo, on state highways in the central coastal region of the state of Yucatán, Mexico. Both records show the importance of studies that quantify the collision of vertebrates on roads to plan its mitigation.

Key words: Conservation, Effect of roads, Felines, Run over.

Uno de los problemas más desafiantes de la conservación biológica, es hacer compatibles las acciones que generan desarrollo económico con la preservación de los ecosistemas naturales y las especies que los habitan (Puc-Sánchez et al. 2013). Un ejemplo claro de esta situación se observa en el desarrollo de la red de carreteras, las cuales son consideradas como pieza clave en el progreso económico y social de cualquier país (Obregón-Biosca 2010). Sin embargo, el desarrollo de estas infraestructuras lineales afecta la supervivencia de la fauna silvestre (Bedoya-V et al. 2018), debido a que representan una 
barrera que impide el movimiento de algunas especies e incrementa la probabilidad de muertes por colisiones con vehículos (Didham et al. 1996; Forman \& Alexander 1998, Bhattacharya et al. 2003; Van der Ree 2009).

Uno de los grupos que se han visto afectados en gran medida por las carreteras son los felinos silvestres (Kerley et al. 2002; Ngoprasert et al. 2007; Ford et al. 2010; Jansen et al. 2010; McGuire 2012; Basille et al. 2013; González-Gallina \& Hidalgo-Mihart 2018). Esto se debe a que suelen tener amplios ámbitos hogareños y recorrer largas distancias (Macdonald et al. 2010). En el estado de Yucatán, habitan 5 de las 6 especies de felinos silvestres presentes en México (Sosa-Escalante et al. 2014), sin embargo, es uno de los estados con menos información de localidades y de sitios de colecta de estos (Escalante et al. 2002).

Al respecto, esta nota contribuye al conocimiento sobre la presencia del Tigrillo y Jaguarundi en la región litoral centro del estado de Yucatán, mediante el registro de dos individuos presuntamente colisionados por vehículos.

El Tigrillo Leopardus wiedii (Schinz 1821) y el Jaguarundi Herpailurus yagouaroundi (É. Geoffroy \& Saint-Hilaire 1803), son felinos neotropicales y simpátricos (De Oliveira 1998a; 1998b). Ambas especies se distribuyen desde el sur de Estados Unidos hasta Uruguay y el norte de Argentina (Emmons \& Feer 1997; Botello et al. 2006). En México, se distribuyen en las llanuras costeras del Pacífico, el Golfo de México y en el centro del país, hacia el Istmo de Tehuantepec y la Península de Yucatán (Hall 1981; Aranda 2005a; Aranda 2005b; MonroyVilchis et al. 2019). Ambas especies se encuentran registradas en el Apéndice I de la Convención sobre el Comercio Internacional de Especies Amenazadas de Flora y Fauna Silvestres (CITES 2019).

El Jaguarundi se encuentra principalmente en bosques tropicales, húmedos y secos, manglares, matorrales y ocasionalmente en bosques de coníferas (De Oliveira 1998b). Es una especie poco conocida con presencia en bajas densidades, se ha mencionado que necesita de cobertura vegetal en buen estado (De Oliveira 1998b; Aranda 2005b; Reid 2009; Almazán-Catalán et al. 2013), aunque también se han registrado cerca y dentro de cultivos (Botello et al. 2013; Farías et al. 2015; Gil-Fernández et al. 2017). En México se encuentra clasificado por la NOM-059-SEMARNAT-2010 (serie de especificaciones técnicas y legales del gobierno mexicano para la protección ambiental de especies nativas de flora y fauna silvestres) como como una especie amenazada (SEMARNAT 2010).

Con relación al Tigrillo, es una especie rara que se encuentra desde las selvas altas, medianas, manglares, bosque mesófilo y en las selvas bajas del Pacífico (DomínguezCastellanos \& Ceballos 2005). Se conoce muy poco de su biología; se sabe que tiene hábitos arborícolas, es nocturno y muy rara vez se le observa durante el día (De Oliveira 1998a; Aranda 2005a). En México está considerado en peligro de extinción, debido a que sus áreas de distribución o el tamaño de sus poblaciones han disminuido drásticamente poniendo en riesgo su viabilidad biológica (SEMARNAT 2010).

El primer evento registrado de manera oportunista fue un individuo de Jaguarundi, el día 13 de enero de 2020 a las 10:41h, que aparentemente murió por atropellamiento (Figura 1), sobre la carretera estatal de dos carriles que comunica el municipio de Dzemul con la localidad de Sacapuc (21.190461, -89.326180; altitud 10 msnm). El individuo no fue colectado ni se pudo determinar su sexo debido al estado de descomposición en el que se encontraba, sin embargo, debido a sus características morfológicas se corroboró que se trataba de un jaguarundi adulto (Aranda 2005b). El paisaje en los alrededores del sitio de 
atropellamiento corresponde a vegetación secundaria de selva baja caducifolia espinosa con cierto grado de perturbación; además, a 400 m aproximadamente se encuentran potreros ganaderos.

El segundo ejemplar registrado de manera casual fue un Tigrillo el día 28 de febrero de 2020 a las 13:55h, que también presentaba características de muerte por atropellamiento, sobre la carretera estatal de dos carriles que comunica el municipio de Dzemul con la localidad de Xtampú (21.277419, -89.321442; altitud 19 msnm; Figura 1) a 9.6 km lineales del registro del Jaguarundi. El individuo no fue colectado, sin embargo, de acuerdo a sus características morfológicas se determinó que era un macho adulto (Aranda 2005a). El paisaje en los alrededores del sitio de atropellamiento fue similar al del jaguarundi. A 3.5 $\mathrm{km}$ al norte del sitio de atropellamiento se encuentra la Reserva Estatal Ciénagas y Manglares de la Costa Norte de Yucatán (RECMY).

Para el estado de Yucatán ya existía un registro previo de un individuo de Jaguarundi atropellado (ver González-Gallina \& Hidalgo-Mihart 2018), sin embargo, no se cuenta con información del sitio de atropellamiento. Para el Tigrillo se cuenta con un registro de atropellamiento en las afueras de la ciudad de Mérida el día 19 de junio del 2020, el cual fue documentado por la prensa digital (yucatanahora.mx).

Los aparentes atropellamientos se presentaron durante el periodo de sequía pre-estival o de primavera, que abarca un periodo de dos a cuatro meses (enero-abril) (Orellana et al. 2009). En esta época, las especies silvestres suelen recorrer grandes distancias cuando el agua escasea, haciéndolos más vulnerables (Williams et al. 2019), lo cual pudiera ser la causa de los probables atropellamientos registrados. Sin embargo, no se descartan otras causas, como el número de vehículos que circulan por la carretera, la cobertura vegetal y la velocidad a la que se desplazan, provocando que los atropellamientos sean más dificiles de evitar (Arroyave et al. 2006).

En los márgenes de las carreteras se observó vegetación secundaria arbustiva y herbácea derivada de la antropización de la selva baja caducifolia, así como la presencia de potreros, los cuales pudieran estar evitando ambas especies debido al riesgo que conlleva cruzar en esos sitios y que es aprovechado por los cazadores (Lira-Torres et al. 2014).

Los registros de atropellamiento son necesarios para identificar las posibles soluciones a la mortalidad de individuos en carreteras (Castilla et al. 2017). Adicionalmente, nuestros registros presentan evidencias sobre la ocurrencia de ambas especies en la región litoral centro del estado de Yucatán, México. Finalmente, es necesario generar información para establecer medidas de mitigación encaminadas a reducir o evitar el atropellamiento de la fauna silvestre, como señales de tránsito para advertir el paso de fauna en algunos puntos de las carreteras, el uso de cercas para impedir el paso de la fauna y la implementación de pasos subterráneos o elevados con las estructuras adecuadas y de diseño específico para las diferentes especies. 

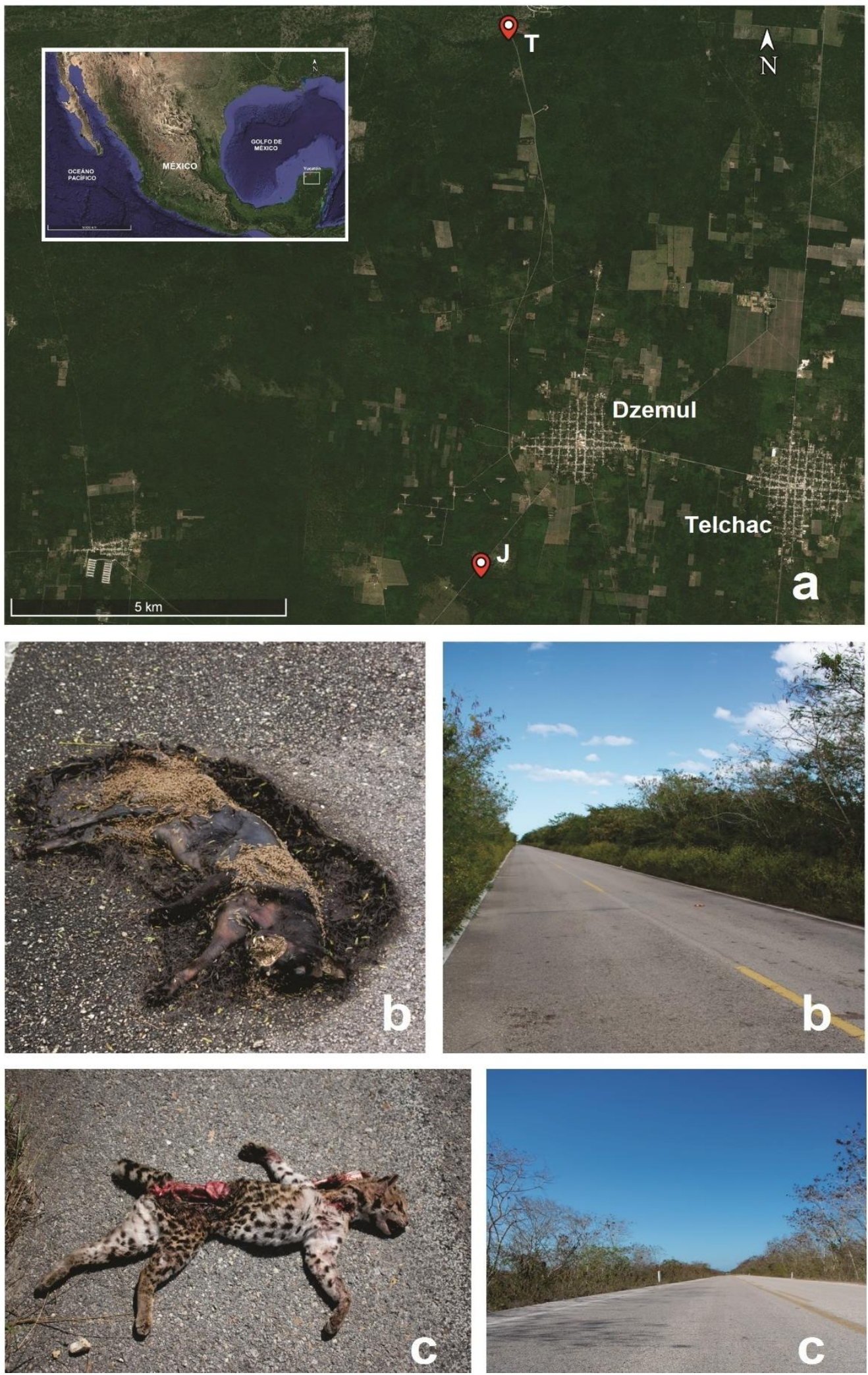

FIGURA 1. a) Localización de los sitios de atropellamiento: I (Jaguarundi), T (Tigrillo). b) Ejemplar de Herpailurus yagouaroundi y vista sur - norte del sitio. c) Ejemplar de Leopardus wiedii y vista sur - norte del sitio. 


\section{AGRADECIMIENTOS}

Los autores agradecen a los revisores anónimos que aportaron valiosos comentarios y sugerencias para mejorar este manuscrito.

\section{REFERENCIAS}

Almazán-Catalán JA, Sánchez-Hernández C, Ruíz-Gutiérrez F, Romero-Almaraz ML, Taboada-Salgado A, Beltrán-Sánchez E, Sánchez-Vázquez L. 2013. Registros adicionales de felinos del estado de Guerrero, México. Revista Mexicana de Biodiversidad 84:347-359. https:// doi.org/10.7550/rmb.23087

Aranda M. 2005a. Leopardus wiedii (Schinz, 1821): Tigrillo. In: Ceballos G, Oliva G, editors. Los Mamíferos Silvestres de México. Fondo de Cultura Económica, Comisión Nacional para el Conocimiento y Uso de la Biodiversidad. p. 361- 362

Aranda M. 2005b. Herpailurus yagouaroundi (Lacépede, 1809): Leoncillo, jaguarundi. In: Ceballos G, Oliva G, editors. Los Mamíferos Silvestres de México. Fondo de Cultura Económica, Comisión Nacional para el Conocimiento y Uso de la Biodiversidad. p. 358-359.

Arroyave M, Gómez C, Gutiérrez M, Múnera D, Zapata P, Vergara I, Andrade L, Ramos K. 2006. Impactos de las carreteras sobre la fauna silvestre y sus principales medidas de manejo. Revista de la Escuela de Ingeniería de Antioquia 5:45-57.

Basille M, Van Moorter B, Herfindal I, Martin J, Linnell JDC, Odden J, Andersen R, Gaillard JM. 2013. Selecting habitat to survive: the impact of road density on survival in a large carnivore. Plos ONE 8: e65493. https://doi.org/10.1371/journal.pone.0065493

Bedoya-V MM, Arias-Alzate A, Delgado-V CA. 2018. Atropellamientos de fauna silvestre en la red vial urbana de cinco ciudades del Valle de Aburrá (Antioquia, Colombia). Caldasia 40(2):335-348.

Bhattacharya M, Primack RB, Gerwein J. 2003. Are roads and railroads barriers to bumblebee movement in a temperate suburban conservation area? Biological Conservation 109(1):3745. https:/ / doi.org/10.1016/S0006-3207(02)00130-1

Botello F, Illoldi-Rangel P, Linaje M, Sánchez-Cordero V. 2006. Primer registro del tigrillo (Leopardus wiedii, schinz 1821) y del gato montés (Lynx rufus, Kerr 1792) en la Reserva de la Biósfera de Tehuacán-Cuicatlán, Oaxaca, México. Acta Zoológica Mexicana (n.s.) 22(1):135-139.

Botello F, Villaseñor E, Guevara L, Méndez A, Cortés A, Iglesias J, Izúcar M, Luna M, Martínez A, Salazar JM. 2013. Registros notables del zorrillo manchado (Spilogale angustifrons) y del jaguarundi (Puma yagouaroundi) en la Reserva de la Biosfera de Tehuacán-Cuicatlán, Oaxaca, México. Revista Mexicana de Biodiversidad 84(2):713-717. https:// doi.org/10.7550/rmb.28873

Castilla MC, Bertucci T, Cuyckens GAE, Díaz MM. 2017. Dos nuevos registros de Leopardus geoffroyi y Puma yagouaroundi (Mammalia: Carnivora: Felidae) en el oeste de la Argentina. Nótulas Faunísticas-Segunda Serie 229:1-5.

CITES. 2019. Convención sobre el comercio internacional de especies amenazadas de fauna y flora silvestres. Apéndices I, II y III. Ginebra, Suiza. https://cites.org/sites/default/files/esp/app/2019/S-Appendices-2019-11-26.pdf. Accessed on 30 june 2020.

De Oliveira TG. 1998a. Leopardus wiedii. Mammalian Species 579:1-6. https:// doi.org/10.2307/3504400 
De Oliveira TG. 1998b. Herpailurus yagouaroundi. Mammalian Species 578:1-6.

https:// doi.org/10.2307/3504500

Didham RK, Ghazoul J, Stork NE, Davis AJ. 1996. Insects in fragmented forests: a functional approach. Trends in Ecology and Evolution 11(6):255-260. https:/ / doi.org/10.1016/0169-5347(96)200473

Domínguez-Castellanos Y, Ceballos G. 2005. Un registro notable del tigrillo (Leopardus wiedii) en la Reserva de la Biosfera Chamela-Cuixmala, Jalisco. Revista Mexicana de Mastozoología 9:146149.

Emmons L, Feer F. 1997. Neotropical rainforest mammals, a field guide. Illinois, Chicago: The University of Chicago Press.

Escalante T, Espinoza D, Morrone JJ. 2002. Patrones de distribución geográfica de los mamíferos terrestres de México. Acta Zoológica Mexicana (n.s.) 87:47-65.

Farías V, Téllez O, Botello F, Hernández O, Berruecos J, Olivares SJ, Hernández-Hernández JC. 2015. Primeros registros de 4 especies de felinos en el sur de Puebla, México. Revista Mexicana de Biodiversidad 86(4):1065-1071. http://dx.doi.org/10.1016/j.rmb.2015.06.014

Ford AT, Clevenger AP, Rettie K. 2010. The Banff wildlife crossings project: An international publicprivate partnership. In: Beckmann JP, Clevenger AP, Huijser M, Hilty JA, editors. Safe passages. Island Press. Washington, U.S.A. p.157-172

Forman RT, Alexander LE. 1998. Roads and their major ecological effects. Annual Review of Ecology, Evolution, and Systematics 29(1):207-231. https://doi.org/10.1146/annurev.ecolsys.29.1.207

Gil-Fernández M, Muench C, Gómez-Hoyos DA, Dueñas A, Escobar-Lasso S, Aguilar-Raya G, Mendoza E. 2017. Wild felid species richness affected by a corridor in the Lacandona forest, Mexico. Animal Biodiversity and Conservation 40.1: 115-120. https://doi.org/10.32800/abc.2017.40.01157

González-Gallina A, Hidalgo-Mihart MG. 2018. A Review of Road-killed Felids in Mexico. Therya 9:147159. http:// dx.doi.org/10.12933/therya-18-584

Hall RE. 1981. The mammals of North America. Weley, Nueva York, Nueva York, EEUU.

Jansen D, Sherwood K, Fleming E. 2010. The I-75 Project: Lessons from the Florida Panther. In: Beckmann JP, editor. Safe passages: highways, wildlife and habitat connectivity. Island Press. Washington, U. S. A. p. 205-221

Kerley LL, Goodrich JM, Miquelle DG, Smirnow E, Quigley HB, Hornocker MG. 2002. Effects of roads and human disturbance on Amur tigers. Conservation Biology 16:97-108. https:// doi.org/10.1046/j.1523-1739.2002.99290.x

Lira-Torres I, Briones-Salas M, Gómez de Anda FR, Ojeda-Ramírez D, Peláez-Acero A. 2014. Acta Zoológica Mexicana (n.s.) 30(1):74-90.

Macdonald DW, Mosser A, Gittleman JL. 2010. Felid society. In: Macdonald DW, Loveridge AJ, editors. Biology and conservation of wild felids. Oxford University Press. Oxford, UK. p. 125-160.

McGuire T. 2012. No ordinary highway: A thirty-year retrospective, Trans-Canada Highway, Banff National Park of Canada. In: Weber S, editor. Rethinking protected areas in a changing world: Proceedings of the 2011 George Wright society conference on parks, protected areas, and cultural sites. The George Wright Society, Hancock, Michigan, USA. p. 197-201.

Monroy-Vilchis O, Zarco-González Z, Zarco-González MM. 2019. Potential distribution and areas for conservation of four wild felid species in Mexico: conservation planning. Mammalian Biology 98:128-136. https://doi.org/10.1016/j.mambio.2019.09.003 
Ngoprasert D, Lynam AJ, Gale GA. 2007. Human disturbance affects habitat use and behavior of Asiatic leopard Panthera pardus in Kaeng Krachan National Park, Thailand. Oryx 41:343-351. https://doi.org/10.1017/S0030605307001102

Obregón-Biosca SA. 2010. Estudio comparativo del impacto en el desarrollo socioeconómico en dos carreteras: Eix Transversal de Catalunya, España, y MEX120, México. Economía, sociedad y territorio 10(32):1-47.

Orellana R, Espadas C, Conde C, Gay C. 2009. Atlas escenarios de cambio climático en la Península de Yucatán. Unidad de Recursos Naturales, Centro de Investigación Científica de Yucatán y Centro de Ciencias de la Atmósfera-UNAM. Mérida, Yucatán, México.

Puc-Sánchez JI, Delgado-Trejo C, Mendoza-Ramírez E, Sauzo-Ortuño I. 2013. Las carreteras como una fuente de mortalidad de fauna silvestre de México. Biodiversitas 111:12-16.

Reid FA. 2009. A field guide to the mammals of Central America and Southeast Mexico. 2d ed. Oxford University Press, New York, US.

SEMARNAT (Secretaría de Medio Ambiente y Recursos Naturales) 2010. Norma Oficial Mexicana NOM059-SEMARNAT-2010, Protección ambiental-Especies nativas de México de flora y fauna silvestres-Categorías de riesgo y especificaciones para su inclusión, exclusión o cambioLista de especies en riesgo. Diario Oficial de la Federación. 30 de diciembre de 2010, Segunda Sección. México.

Sosa-Escalante JE, Hernández-Betancourt S, Pech-Canché JM, MacSwiney MC, Díaz-Gamboa R. 2014. Los mamíferos del estado de Yucatán. Revista Mexicana de Mastozoología Nueva época 1(4):40-59.

Van der Ree R. 2009. The ecology of roads in urban and urbanizing landscapes. In: McDonnell MJ, Hahs AK, Breuste JH, editors. Ecology of cities and Towns: A comparative approach. Cambridge, University Press. p. 185-196.

Williams ST, Collinson W, Patterson-Abrolat C, Marneweck DG, Swanepoel L. 2019. Using road patrol data to identify factors associated with carnivore roadkill counts. PeerJ 7:e6650 http:// doi.org/10.7717/ peerj.6650

yucatanahora.mx. 2020. https://yucatanahora.mx/un-tigrillo-se-acerco-a-los-humanos-y-murioatropellado/?fbclid=IwAROC_uqNDgLaGTpV9GXjE3GItTF80GzgmBRJ06-

Hx1HWgleRnqfNkPW2OwY Accessed on 07 August 2020.

Editor: José F. González-Maya

Recibido: 2020-07-08

Revisado: 2020-08-20

Aceptado: 2020-09-03

Publicado: 2020-10-13 